\title{
Output Delay Sliding Mode Tracking Control of SRM based on Multi-innovation Model Identification
}

\author{
Lei Yang ${ }^{1}$, Chuansheng Tang ${ }^{1, *}$, Jie Yang ${ }^{2}$, Yongxin $\mathrm{Li}^{3}$ and ${\mathrm{Tao} \mathrm{Li}^{4}}^{4}$ \\ ${ }^{1}$ Nanyang Institute of Technology, Nanyang Henan 473004, China \\ ${ }^{2}$ Henan Institute of Technology, Xinxiang Henan 453000, China \\ ${ }^{3}$ Xinxiang University, Xinxiang Henan 453003, China \\ ${ }^{4}$ Department of Informatics, University of Zurich, Zurich 8050, Switzerland
}

Received 9 May 2020; Accepted 14 September 2020

\begin{abstract}
The drive system of switched reluctance motor (SRM) is a complex nonlinear system that is composed of many links. The delay in the measurement of the speed and position signal of SRM is caused by the factors that affect the measuring sensor. To effectively improve the influence of the SRM rotor position and speed signal delay on the system performance, a sliding mode position tracking method based on output delay observation was proposed in this study. First, the model was discretized according to the structure and characteristics of SRM and the mathematical parameters of the system were identified using a multi-innovation stochastic gradient (MISG) algorithm. Second, a delay state observer was constructed on the basis of an SRM system model with output delay. Then, the sliding mode tracking control method based on the delay state observation compensation was proposed and combined with sliding mode control theory. Lastly, the effectiveness of the designed model parameter identification, delay state observation, and output delay control methods were compared through numerical simulation. Results show that when uncertain factors, such as noise, are present in the system, the MISG identification method can rapidly and accurately identify the parameters of the SRM model compared with the stochastic gradient identification method; the identification accuracy of the former is four times higher than that of the latter. Similarly, the sliding mode position tracking control method based on output delay observer can rapidly and accurately track the position and speed within $0.5 \mathrm{~s}$. However, its position $(0.2 \mathrm{rad})$ and velocity $(0.233$ $\mathrm{rad} / \mathrm{s}$ ) tracking exhibit large steady-state errors when no delay observation compensation is present. The proposed method not only demonstrates high position tracking accuracy, but also possesses strong robustness to output delay.
\end{abstract}

Keywords: SRM, Model identification, Sliding mode control, Output delay

\section{Introduction}

Switched reluctance motor (SRM) is a doubly salient variable reluctance motor that works in a continuous switch mode. The stator pole of an SRM is surrounded by concentrated winding, and the rotor is made of silicon steel sheet with high permeability. The drive system of this motor consists of four parts, namely, SRM, power converter, controller, and detector, and its system follows the principle of minimum magnetoresistance in operation to ensure that the flux is always closed along the path of minimum reluctance. Therefore, SRM is one of the most potential, efficient and energy-saving variable speed motor drive systems. Emerson Electric Co., a multinational motor company, regarded SRM as a new technology and economic growth point of speed control drive systems in the 21 st century. SRM has simple and solid structure, wide speed range, excellent speed performance, high efficiency in the entire speed range, and high system reliability. This motor is widely used in electric vehicle drives, household appliances, general industry, aviation industry, and servo systems. In addition, SRM covers all kinds of high- and low-speed drive systems with a power range of $10 \mathrm{~W}-5 \mathrm{MW}$ and therefore presents a strong market field potential [1-2]. The rotors of SRMs used in electric vehicles do not contain permanent

\footnotetext{
*E-mail address: tes111@163.com

ISSN: $1791-2377$ @ 2020 School of Science, IHU. All rights reserved.

doi:10.25103/jestr.135.15
}

magnets and only consist of low-loss silicon steel and stator winding, which reduce the manufacturing cost and help maintain good mechanical and thermal stabilities; such features are beneficial to power vehicles [3].

Although the electromagnetic principle and structure of SRM are simple, the magnetic circuit of the motor changes periodically and experiences serious local saturation. The drive system of SRM involves many disciplines, such as motor, power electronics, microelectronics, computer control, and mechanical dynamics, and its design, performance analysis, and control are more difficult than those of traditional motors. On the one hand, the nonlinearities of the internal magnetic field, nonlinear switching power supply, and phase current waveform in SRM are difficult to analyze. Therefore, this study explores the analysis and methods for the accurate calculation of the electromagnetic torque of SRMs [4-5]. On the other hand, accurately establishing the dynamic model of the SRM drive system is difficult. The model is nonlinear, multivariable, and multiparameter and possesses strong coupling, which increases the difficulty of system control [6].

The SRM drive system is a complex nonlinear system composed of a power converter, controller, and detector. In actual motion, the delay in the measurement of the speed and position signals of SRM is caused by the factors that affect the measuring sensors, which reduce the tracking performance of the system [7]. Moreover, because of the 
complexity of the working environment, the parameter uncertainty, load disturbance, thrust fluctuation, and friction in the SRM system make the design of control system difficult [8]. Therefore, the multifactor-constrained control of SRM under output delay condition is practical but challenging.

\section{State of the art}

To address the problem in the establishment of the motor model, various identification methods are adopted to identify the motor parameters [9-14]. Odhano et al. [9] systematically reviewed the parameter identification methods of induction, synchronous, and synchronous reluctance motors. However, they failed to provide the specific identification methods and processes. Through the mathematical model of the permanent magnet synchronous motor (PMSM) system, Xu et al. [10] constructed the linear regression model of the SRM system and applied the forgetting factor random gradient algorithm to identify the system parameters. The simulation results showed that this algorithm has better advantages than the stochastic gradient (SG) algorithm in terms of the convergence speed and accuracy of non-sensitive output parameter identification. However, the former only used the input and output information of the current time in the identification process, and the convergence speed and accuracy requires further improvement. To improve the calculation accuracy and evaluation ability of the motor system's energy consumption model, Qu et al. [11] proposed the energy consumption correction method of the clustered motor system on the basis of the parameter identification of the Levenberg-Marquardt algorithm. This method performed parameter identification based on the current input and output. However, its convergence speed and identification accuracy were limited. Accetta et al. [12] transformed the offline identification of the linear induction motor model parameters into an optimization problem and adopted genetic algorithm for the identification and optimization processes. The identification accuracy of this method depended on the size of the initial population. Fagiano et al. [13] studied the estimation of the induction motor model parameters by measuring the data from circuit breakers equipped with industrial sensors. The derivative of the three-phase stator voltage and current was obtained using the circuit breaker, which was then used to establish the identification problem based on optimization. On the one hand, the introduction of circuit breakers reduced the reliability of the system. On the other hand, the delay in data acquisition affected the accuracy of the identification. Zhang et al. [14] introduced the variable step size adaptive linear neural network into the parameter identification of PMSM and designed an intelligent parameter identification method to improve the convergence speed and reduce the corresponding steady-state error. The identification accuracy of this method depended on the structure and data scale of the neural network, and the complex structure and large data reduced the convergence speed of identification. Scholars rarely investigated the delay problem in motors [15-16]. In their study on the traction drive system of rail transit, Wang et al. [15] analyzed the causes and effects of control delay on the basis of the operational characteristics of high-power permanent magnet synchronous traction motor and designed a delay compensation method based on the high-sampling rate observer. The experimental results showed that the designed compensation method accelerated the dynamic response of the current control and reduced the steady-state current ripple. In addition, the accuracy of the observer depended on the model parameters of the system. The imprecise flux observation model of the induction motor and the cross-coupling between two currents in the current loop due to control delay resulted in the current distortion and instability of the drive system with low switching frequency. Pan et al. [16] applied neutral theory, proposed a current decoupling control method for induction motors based on neutral type, and designed a neutral current controller. The neutral current decoupling control method eliminated the influence of digital delay on the control performance of the transmission system by establishing an accurate mathematical model. However, the accurate model parameters were still based on the system model parameters, which hardly met the performance requirements of highprecision systems. The complex factors in SRM systems, such as strong coupling, nonlinearity, and multiple timevarying, must be addressed to achieve the high-performance dynamic control of the SRM drive system [17-26]. Jeon et al. [17] controlled the magnetic field of the electromagnet using a fixed gain proportional-integral-derivative (PID) controller to obtain a constant current output. However, when the internal characteristics of the system changed or the external disturbance greatly varied with the fixed control parameters, the performance of such parameters remarkably declined and caused system instability and collapse. Therefore, Angel et al. [18] introduced a fractional order operator into the PID control and increased the design freedom. The proposed fractional order PID controller was robust to parameter changes, and the presented method displayed robustness to small-scale disturbances. However, the application of the proposed method was limited. Adaptive control identified the system model parameters online, but this method was still based on the system model, and its online parameter estimation not only increased the calculation of the system, but also reduced the dynamic response ability of the system [19]. Tang et al. [20] estimated the stator current of linear motors online using a sliding mode observer in the $\alpha-\beta$ static coordinate system and the position and speed of the actuator through a back electromotive force model. This method effectively avoided the influence of external disturbance on the speed and position estimation accuracy of the actuator but exhibited a strong dependence on the stator resistance. To improve the chattering phenomenon caused by the traditional sliding mode control, Nihad et al. [21] designed a load disturbance observer, which effectively improved the dynamic performance of the motor system. Despite achieving a high estimation accuracy in high-speed operations, the application of this method is limited due to its large error in low-speed ones. Abdelkader et al. [22] combined adaptive and backstep controls to reverse design an uncertain DC motor speed control system. However, this approach required numerous derivations of the system model, which led to a calculation explosion problem. To improve its performance, this technique should be combined with other methods. Tang et al. [23] developed the integrated design of the speed, thrust, and flux loops using an adaptive backstepping control, which, however, resulted in computational explosion. Mohamed et al. [24] applied predictive control to an induction motor and proposed a sensorless direct torque predictive control method. This method utilized the extended Kalman filter as the driver to estimate the state of the motor model. Although this approach effectively reduced the flux and torque ripple, the prediction of the system state variables 
requires the previous and model parameter data, and the size of the predictive control sample data exerted a large effect on the system accuracy. Masoudi et al. [25] used fuzzy estimation to realize the automatic gain adjustment of sliding mode control. This method, which combined the selflearning ability of the fuzzy control and the insensitivity of the sliding mode control with the time-varying parameters, resolved the chattering phenomenon of the system to some extent. However, such phenomenon was not eliminated and still affected the performance of the system. To reduce the chattering caused by the sliding mode control, Tang et al. [26] improved the dynamic performance of the system by introducing a nonlinear disturbance observer to observe and compensate the unmodeled dynamic and external disturbances. However, the accuracy of the observer was dependent on the model parameters of the system, hence, satisfying the requirements of high-accuracy events was difficult.

Regardless of the type of control (e.g., position, speed, or current control), the total amount of the control input of an actual motor system is not infinite, and thus should be designed under a rated state (e.g., rated torque, rated voltage, and rated current). The abovementioned control methods rarely considered the saturation limit. The existence of control input saturation constraints inevitably affects the dynamic performance of the system. Therefore, the multifactor constraint control method of SRM that involves control input saturation constraints should be further investigated.

This study proposed a sliding mode position tracking method for SRM on the basis of an output delay observer. The SRM model parameters were identified using the multiinnovation stochastic gradient (MISG) method. The proposed approach considered the restriction of the sensor detection's output delay condition, and an output delay observer was designed to observe and compensate for the rotor position and speed of SRM. The sigmoid function was adopted to improve the chattering phenomenon of the sliding mode control, improve the robustness and tracking ability of the system, and realize the high-performance position tracking control of SRM.

The remainder of this study is organized as follows. Section 3 describes the SRM structure, constructs the corresponding dynamic and discrete digital models, designs the output delay observer and fuzzy position controller, and presents the stability analysis. Section 4 compares the SG identification technique and sliding mode control method without delay observation compensation using numerical simulation in MATLAB and subsequently verifies the validity and superiority of the proposed method. Lastly, section 5 provides the conclusions.

\section{Methodology}

\subsection{Structure and mathematical model of SRM}

Fig. 1 shows a three-phase SRM, which consists of six stator poles and four rotor poles. The stator has a concentrated winding, whereas the rotor has no winding.

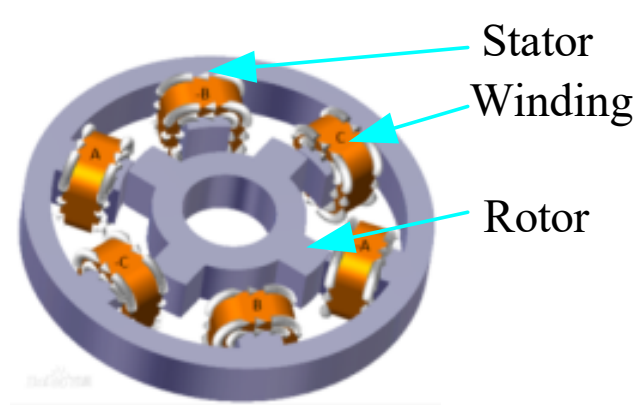

Fig. 1. Structural model of the SRM

The influences of core loss and mutual inductance are disregarded, and the main circuit voltage and motor speed are assumed to be constant and periodically constant, respectively. The physical model of SRM can be mathematically expressed as:

$$
\left\{\begin{array}{l}
U_{k}=R_{k} i_{k}+d \psi_{k} / d t, k=a, b, c \\
T_{m}=\sum_{k=1}^{3} \frac{\partial \lambda_{k}(x, i)}{\partial x} i_{k} \\
T_{m}=J_{n} \ddot{\theta}+B_{n} \dot{\theta}+T_{L}+T_{f}+T_{r}
\end{array}\right.
$$

where $U_{k}, i_{k}, \psi_{k}$, and $R_{k}$ represent the voltage, current, flux, and resistance of $k$-phase winding, respectively; $T_{m}$ represents the electromagnetic torque; $J_{n}$ denotes the mass of the rotor; $\theta$ represents the position of the rotor; $\dot{\theta}=\omega$ is the angular speed of the rotor; $B_{n}$ is the linear friction coefficient; and $T_{r}, T_{L}$, and $T_{f}$ are the torque fluctuation, load disturbance, and nonlinear friction torque of the system, respectively.

The transfer function from the electromagnetic torque of the system to the rotor position is defined as:

$$
G(s)=\frac{1}{J_{n} s^{2}+B_{n} s}
$$

\subsection{Multi-innovation parameter identification of the} SRM model

The SRM model can be discretized as:

$$
\begin{aligned}
y(k) & =-\operatorname{den}(2) y(k-1)-\operatorname{den}(3) y(k-2) \\
& +\operatorname{num}(2) u(k-1)+\operatorname{num}(3) u(k-1)
\end{aligned}
$$

The parameter vectors are defined as:

$$
\begin{aligned}
\theta(k) & =\left[\begin{array}{llll}
a_{1}(k) & a_{2}(k) & b_{1}(k) & b_{2}(k)
\end{array}\right]^{T} \\
& =\left[\begin{array}{llll}
\operatorname{den}(2) & \operatorname{den}(3) & \operatorname{num}(2) & \operatorname{num}(3)
\end{array}\right]^{T}
\end{aligned}
$$

And the innovation vectors are expressed as:

$$
\psi^{T}(k)=\left[\begin{array}{llll}
-y(k-1) & -y(k-2) & u(k-1) & u(k-2)
\end{array}\right]^{T}
$$

Eq. (2) can be written as:

$$
y(k)=\psi^{T}(k) \theta(k)
$$


Considering the influence of measurement noise on the system and assuming that the mean value of a random noise is zero, the identification model can be defined as:

$$
y(k)=\psi^{T}(k) \theta(k)+v(k)
$$

The MISG algorithm is used to identify the parameters of Eq. (7) online, which lay the foundation for the subsequent design of the observer and controller. The MISG method is an extension of the SG identification algorithm. The SG parameter identification process is mathematically expressed as:

$$
\left\{\begin{array}{l}
\hat{\theta}(k)=\hat{\theta}(k-1)+\frac{\psi(k)}{r(k)} e(k), \hat{\theta}(0)=\frac{1_{n}}{p_{0}} \\
e(k)=y(k)-\psi^{T}(k) \hat{\theta}(k-1) \\
r(k)=r(k-1)+\|\psi(k)\|^{2}
\end{array}\right.
$$

where $1_{n}=[1,1, \cdots, 1]^{T} \in R^{n}$ is an $n$-dimensional vector and $p_{0}$ is a large positive number $\left(p_{0}=10^{6}\right)$. Eq. (8) suggests that this method only uses the current innovation data in parameter estimation. The MISG identification method introduces the innovation length $p$, which not only uses the current identification innovation, but also utilizes the previous one to improve the convergence speed and identification accuracy. The specific process can be mathematically expressed as:

$$
\left\{\begin{array}{l}
\hat{\theta}(k)=\hat{\theta}(k-1)+\frac{\Psi(p, k)}{r(k)} \mathrm{E}(p, k), \hat{\theta}(0)=\frac{1_{n}}{p_{0}} \\
\mathrm{E}(p, k)=Y(p, k)-\Psi^{T}(p, k) \hat{\theta}(k-1) \\
r(k)=r(k-1)+\|\Psi(p, k)\|^{2}, r(0)=1 \\
Y(p, k)=[y(k), y(k-1), \cdots, y(k-p+1)]^{T} \\
\Psi(p, k)=[\psi(k), \psi(k-1), \cdots, \psi(k-p+1)]^{T}
\end{array}\right.
$$

The multi-innovation gradient criterion function is written as:

$$
J(\theta):=\left\|Y(p, t)-\Psi^{T}(p, t) \theta\right\|^{2}
$$
2.

The flow chart of the MISG method is illustrated in Fig.

MATLAB is used to transform the identified model parameters into the model parameters of Eq. (2) to determine the model parameters $\left(J_{n}\right.$ and $\left.B_{n}\right)$ of SRM.

\subsection{Design of the output delay observer}

Lemma 1 [27]. The linear delay system is defined as:

$$
\dot{z}(t)=A z(t)+B z(t-\tau)
$$

where $z=\left[\begin{array}{llll}z_{1} & z_{2} & \cdots & z_{n}\end{array}\right]^{T}, A \in R^{n \times n}$ is an $n$-dimensional real matrix, $B \in R^{m \times n}$ is a real matrix, and $\tau$ is a time delay constant. The condition for the stability of the system is expressed as:

$$
s I-A-B e^{-\tau s}=0
$$

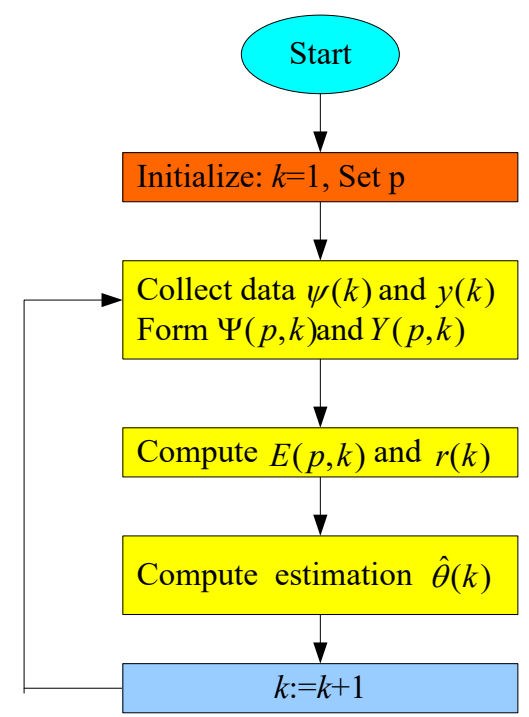

Fig. 2. MISG identification of the SRM parameters

The real parts of the corresponding eigenvalues are negative, and the delay system is exponentially stable. In Eq. (12), $I \in R^{n \times n}$ is the unit matrix.

If $x_{1}=\theta$ and $x_{2}=\dot{x}_{1}=\dot{\theta}$, Eq. (2) can be written as:

$\dot{x}=\left[\begin{array}{ll}\dot{x}_{1} & \dot{x}_{2}\end{array}\right]^{T}=A x+B u$

where $A=\left[\begin{array}{cc}0 & 1 \\ 0 & -a\end{array}\right], \quad B=\left[\begin{array}{l}0 \\ b\end{array}\right], \quad b=B_{n} / J_{n}, \quad$ and $a=-B_{n} / J_{n}$.

Assuming that the output signal experiences delay and given that $\tau$ is the output position delay constant, the actual output can be expressed as:

$$
y(t)=\theta(t-\tau)=C x(t-\tau)=\left[\begin{array}{ll}
1 & 0
\end{array}\right] x(t-\tau)
$$

The objective of the observation is $\hat{\theta}(t) \rightarrow \theta(t)$ when $t \rightarrow \infty$.

For the delay system composed of Eq. (13) and Eq. (14), a simple linear delay observer can be designed as:

$$
\dot{\hat{z}}(t)=A \dot{z}(t)+B u(t)+K[y(t)-C z(t-\tau)]
$$

where $\hat{z}(t)$ is the estimated signal of $z(t)$ and $\hat{z}(t-\tau)$ is the delay signal of $\hat{z}(t)$.

If the delay estimation error is determined as $\tilde{z}(t)=z(t)-\hat{z}(t)$, then

$$
\tilde{z}(t)=A z(t)-K C Z(t-\tau)
$$

According to Lemma 1, the delay system is asymptotically exponentially stable if the real part of the eigenvalue in Eq. (16) becomes negative after selecting the appropriate control gain $K$, that is, the exponent of $z(t)$ converges to zero when $t \rightarrow \infty$. 
3.4 Design of the sliding mode controller and stability analysis

As previously established, this study focuses on position tracking control. Hence, the system can be mathematically written as:

$$
\ddot{\theta}=-a \dot{\theta}+b u+d, u=T_{m}
$$

where $u=T_{m}$ is the system control input and $d t=T_{L}+T_{f}+T_{r}$ is the total uncertainty of the system. In addition, the system satisfies the condition $\|d t\| \leq D$.

The control objective is that the designed input is $u$ when Eq. (17) exists the output delay, so that the system can achieve the desired position tracking $\theta_{d}$ and the desired angular speed tracking $\omega_{d}=\dot{\theta}_{d}$ of the motor rotor, that is, $\theta \rightarrow \theta_{d}$ and $\omega \rightarrow \omega_{d}$ when $t \rightarrow \infty$. The sliding surface function is designed as follows:

$$
s=c e+\dot{e}, c>0, e=\theta_{d}-\theta
$$

The control rate in Eq. (19) and the observer in Eq. (15) are used in Eq. (17). The asymptotically stable system structure is presented in Fig. 3.

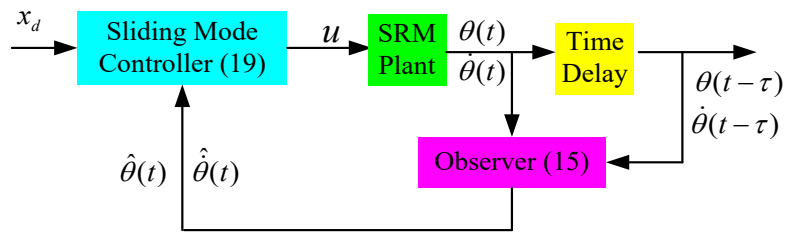

Fig. 3. Sliding mode controller of the SRM based on delay observer

$$
\begin{aligned}
& u=\frac{1}{\mathrm{~b}}\left(\ddot{\theta}_{d}+a \dot{\hat{\theta}}+\eta \hat{s}+c \dot{\hat{e}}+\xi \operatorname{sign}(s)\right) \\
& \eta>\max (\mathrm{D}, 1), \hat{e}=\theta_{d}-\hat{\theta}, \hat{s}=c \hat{e}+\hat{\dot{e}}
\end{aligned}
$$

where $\tilde{\theta}=\theta-\theta_{d}, \tilde{\dot{\theta}}=\dot{\theta}-\hat{\dot{\theta}}, \tilde{e}=e-\hat{e}=-\theta+\hat{\theta}=-\tilde{\theta}$, $\tilde{\dot{e}}=-\tilde{\dot{\theta}}, \tilde{s}=s-\widehat{s}=c \tilde{e}+\tilde{\dot{e}}=-c \tilde{\theta}-\tilde{\dot{\theta}}$, and $\eta>1$.

Given the Lyapunov function $V=\frac{1}{2} s^{2}$, the derivative of the function along the Eq. (13) can be obtained as $\dot{V}=s \dot{s}=-\eta s^{2}-\eta c \tilde{\theta} s+(a-\eta-c) \dot{\tilde{\theta}} s-\xi|s|=-\eta s^{2}-k_{1} s \tilde{\theta}+k_{2} s \dot{\tilde{\theta}}-\xi|s|$ , where $k_{1}=\eta c$ and $k_{2}=(a-\eta-c)$. Considering that $k_{1} s \tilde{\theta} \leq \frac{1}{2} s^{2}+\frac{1}{2} k_{1}^{2} \tilde{\theta}^{2} \quad$ and $\quad k_{2} s \tilde{\dot{\theta}} \leq \frac{1}{2} s^{2}+\frac{1}{2} k_{2}^{2} \tilde{\dot{\theta}}^{2} \quad$, $\dot{V} \leq-(\eta-1) s^{2}-\eta c \tilde{\theta} s+\frac{1}{2} k_{1}^{2} \tilde{\theta}^{2}+\frac{1}{2} k_{2}^{2} \tilde{\dot{\theta}}^{2}$.

Given that the observer is exponentially convergent and $\eta>\max (\mathrm{D}, 1) \quad$ a $\quad \eta_{1}=\eta-1>0 \quad$, then $\dot{V} \leq-\eta_{1} V+\chi(\cdot) e^{-\sigma_{0}\left(t-t_{0}\right)} \leq-\eta_{1} V+\chi(\cdot), \sigma_{0}>0$, where $\chi(\cdot)$ is a class $K$ function of $\left\|\tilde{x}\left(t_{0}\right)\right\|$ and $x=\left[\begin{array}{ll}\theta & \dot{\theta}\end{array}\right]$.

Lemma 2 [27]. For $V \in[0, \infty]$, the solution for the inequality equation $\dot{V} \leq-\alpha V+f, \forall t \geq t_{0} \geq 0 \quad$ is
$V(t) \leq e^{-\alpha\left(t-t_{0}\right)} V\left(t_{0}\right)+\int_{t_{0}}^{t} e^{-\alpha\left(t-t_{0}\right)} f(\varsigma) d \varsigma$, where $\alpha$ is any constant.

According to Lemma 2, $V(t) \leq e^{-\eta_{1}\left(t-t_{0}\right)} V\left(t_{0}\right)+\frac{\chi(\cdot)}{\eta_{1}}\left(1-e^{\eta_{1}\left(t-t_{0}\right)}\right) \quad, \quad$ in which $\lim _{t \rightarrow \infty} V(t) \leq \frac{1}{\eta_{1}} \chi(\cdot)$ and $V(t)$ is asymptotically stable and has a stability accuracy that depends on $\eta_{1}$.

To address the chattering phenomenon caused by the switching function of the conventional sliding mode control, the sigmoid function is used instead of the switching function. The control input of the system is expressed as:

$$
u=\frac{1}{b}\left(\ddot{\theta}_{d}+a \dot{\hat{\theta}}+\eta \hat{s}+c \dot{\hat{e}}+\xi \gamma(s)\right)
$$

And the sigmoid function is defined as:

$$
\gamma(s)=\frac{2}{(1+\exp (-\rho s))}-1, \rho>0 .
$$

\section{Results and discussion}

The proposed method is analyzed in terms of system identification, observation performance, and control effect.

The nominal parameters of the SRM model include the rotor moment of inertia $J_{n}=8 \times 10^{-3} \mathrm{~kg} \cdot \mathrm{m}^{2}$, friction coefficient $B_{n}=0.2 \mathrm{~N} \cdot \mathrm{m} / \mathrm{s}$, total system disturbance $d t=10 \sin t, \quad f(x, t)=-a x_{2}, \quad a=B_{n} / J_{n}=25, \quad$ and $b=1 / J_{n}=125$. The system model can be expressed as:

$\left\{\begin{array}{l}\dot{x}_{1}=x_{2} \\ \dot{x}_{2}=-25 x_{2}+125 u+d t\end{array}\right.$

The sampling period of the system is $T_{s}=1 \mathrm{~ms}$, and the command signal of the rotor position is $x_{1}=x_{d}=\sin t$. Assuming that the delay constant of the system is $\tau=0.2 \mathrm{~s}$, Eq. (22) is discretized and the influence of the external disturbance is disregarded when identifying the parameters. The discretized model is written as:

$$
\begin{aligned}
y(k)= & 1.9753 y(k-1)+0.9753 y(k-2) \\
& +6.1982 \times 10^{-5} u(k-1)+6.1468 \times 10^{-5} u(k-2)
\end{aligned}
$$

\subsection{System model parameter identification}

Considering the influence of measurement noise and other factors during identification, Eq. (23) can be expressed as:

$$
y(k)=\Phi^{T} \theta+v(k)
$$

where $\psi^{T}(k)=\left[\begin{array}{llll}-y(k-1) & -y(k-2) & u(k-1) & u(k-2)\end{array}\right]^{T}$ is the innovation vector, $\theta(k)=\left[\begin{array}{llll}a_{1}(k) & a_{2}(k) & b_{1}(k) & b_{2}(k)\end{array}\right]^{T} \quad$ is the parameter vector with a true value of $\theta_{d}=\left[\begin{array}{llll}-1.9753 & 0.9753 & 6.1982 \times 10^{-5} & 6.1468 \times 10^{-5}\end{array}\right]^{T}$, 
and $v(k)$ has a zero mean. In addition, $\sigma=0.5$ is the uniformly distributed white noise, and the signal-to-noise ratio is $\delta_{m s}=14.45 \%$. The system parameters are subsequently identified using the SG and MISG methods as shown in Fig. 4.

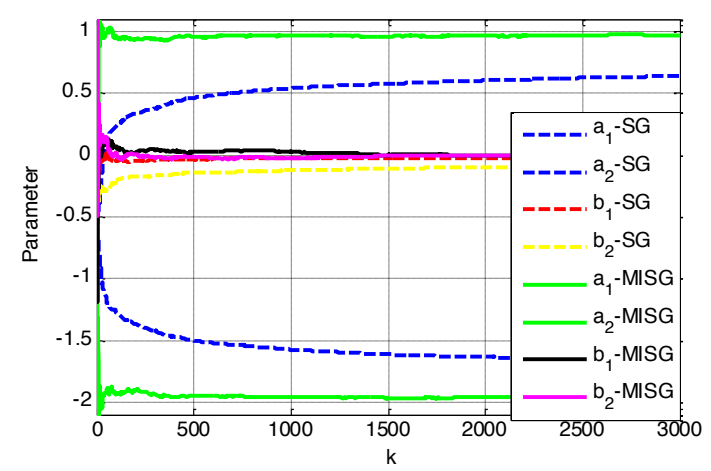

(a) Parameter estimation curve of SRM

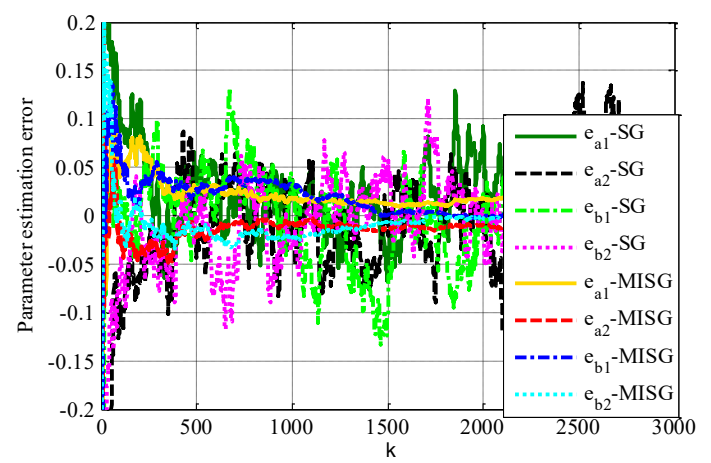

(b) Parameter estimation error curve of SRM

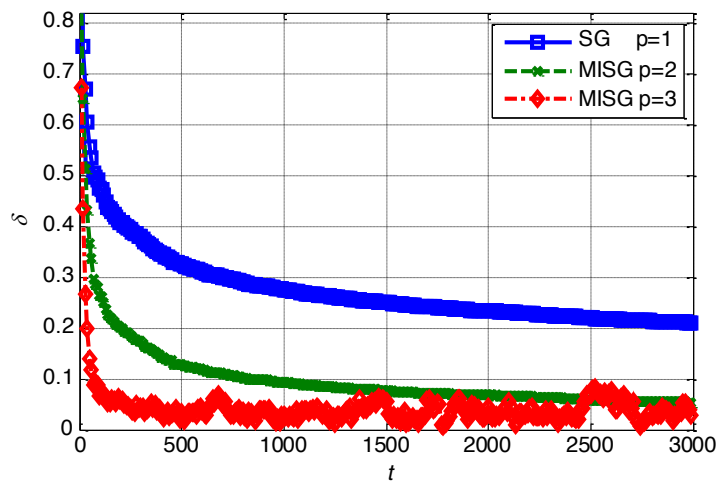

(c) Quantitative error curve for the parameter estimation of SRM

Fig. 4. Parameter identification response curves of SRM

Fig. 4 shows that when noise interference is present, although the calculation of the SG identification is small, the identification accuracy is low because the algorithm only uses the current innovation data in parameter estimation. Conversely, the MISG algorithm uses the previous and current identification information and therefore improves the identification accuracy. The value of $a_{1}$ reaches -1.970 when $k=3000$, the estimation error is only 0.0053 , and the random gradient algorithm error is 0.3068 . To compare the influences of different dynamic information lengths on the system performance, the quantization error of parameter estimation $\delta=\|\hat{\theta}(k)-\theta(k)\| /\|\theta(k)\|$ is introduced. Fig. 4(c) shows that the system identification accuracy rapidly increases with the increase in the dynamic information length $p$; the random gradient method is a special case of $p=1$. After 3000 iterations, the system parameters obtained through MISG identification are expressed as $\theta(3000)=\left[\begin{array}{llll}-1.970 & 0.9725 & -5.276 \times 10^{-3} & 4.79 \times 10^{-3}\end{array}\right]^{T}$

. This finding suggests that small parameter identification has a larger deviation than the expected value. However, the error is within a range of six thousandths, which satisfies the actual needs.

\subsection{Analysis of the performance of the output delay observer}

The analysis of the performance of the output delay observer is illustrated in Fig. 5.
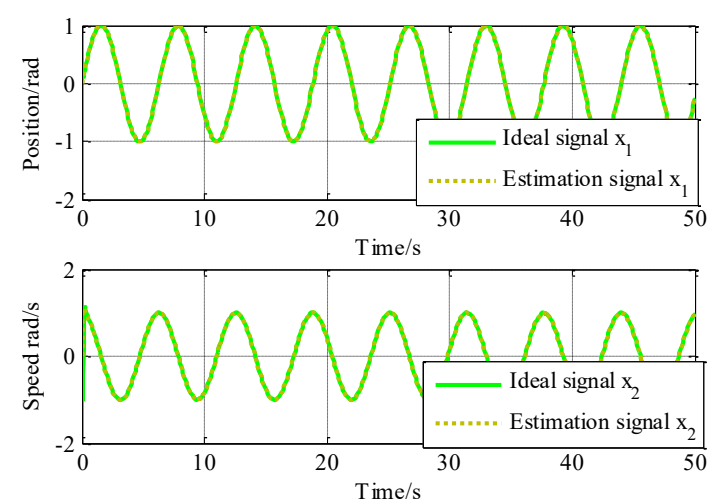

(a) Rotor position and speed curve
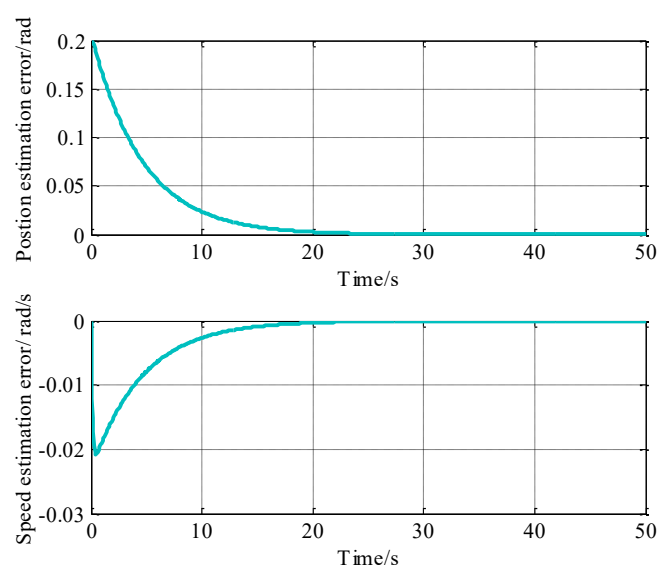

(b) Rotor position and speed output delay observation response curve of SRM

Fig. 5. Observation response curves of the rotor position and speed output delay

Fig. 5 indicates that when a delay output exists in the system, the proposed delay observer can fully track the rotor position and speed of SRM within 20s, and the corresponding estimation accuracy and dynamic response ability are high.

\subsection{Analysis of the performance of the controller}

The sliding mode control method based on output delay observer is compared with the method version without observation. The simulation results are presented in Fig. 6 .

Fig. 6 shows that when output delay is present in the system, the sliding mode control with delay observer can rapidly track the speed and position within $0.5 \mathrm{~s}$. Without delay observation compensation, the system obtained constant position and speed errors of $0.2 \mathrm{rad}$ and $0.233 \mathrm{rad} / \mathrm{s}$, respectively. As shown in Fig. 6(c), the sliding mode control 
with sigmoid function has a smooth control input, which is convenient for practical engineering applications.
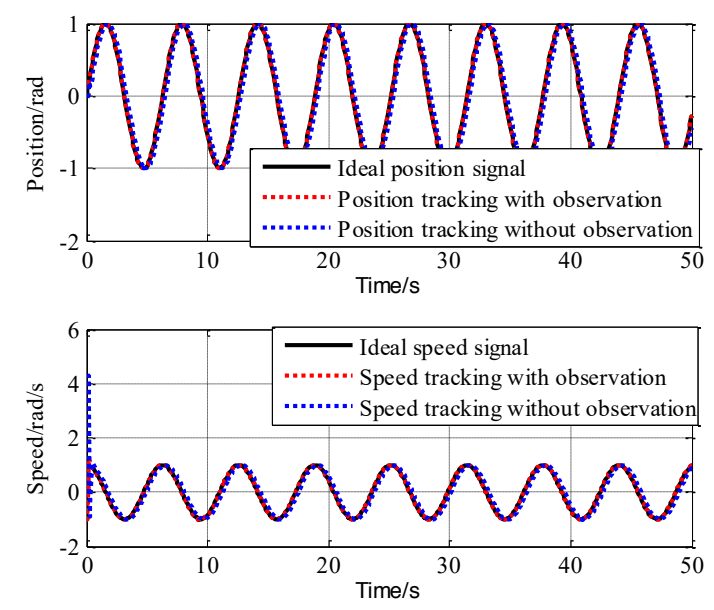

(a) Rotor position and speed tracking curve
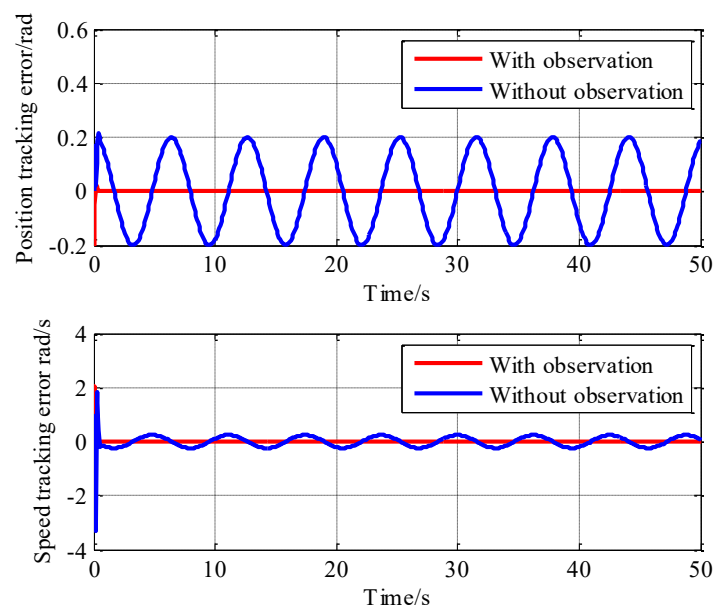

(b) Motor rotor position and speed tracking error curve

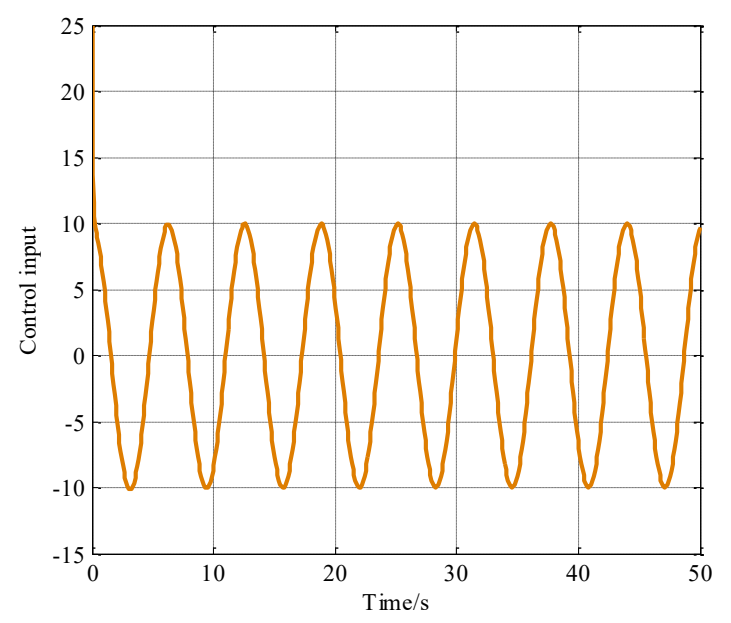

(c) Control input response curve

Fig. 6. Response curves of the SRM control system

\section{Conclusions}

To investigate the influence of output delay factors on the control system accuracy, this study constructed the mathematical model of SRM, combined system identification, observer design, and sliding mode control theories and designed a new position tracking method for SRM by combining theoretical derivation and numerical simulation. The following conclusions could be drawn as follows:

(1) Under the influence of random noise in the system, the introduction of multi-innovation length can effectively improve the identification accuracy of the system parameters. The MISG method $(p=3)$ has a higher identification accuracy than the $\mathrm{SG}$ approach $(p=1)$. Moreover, the quantization error of parameter estimation is reduced by four times (from 0.2 to 0.05 ), and the identification accuracy increased by four times.

(2) When output delay is present in the system, the designed simple feedback state observer rapidly and accurately tracks the rotor position and speed of SRM, thereby realizing the observation and compensation of the output. Consequently, the system achieves an error-free signal tracking within $20 \mathrm{~s}$.

(3) When output delay and external disturbance are present, the proposed method rapidly tracks the position and speed of the SRM rotor within $1.2 \mathrm{~s}$. However, its position and speed tracking incur a steady-state error when no observer compensation exists.

(4) The sigmoid function in the controller leads to a smooth control input curve and effectively resolves the chattering phenomenon of the sliding mode control.

This study, which is mainly based on the structural model of SRM, combines theoretical analysis and numerical simulation. The proposed method effectively improves the output delay of the system and satisfies the requirements of high-speed and high-precision servo systems. However, this approach requires further verification and optimization through experiments to address the limited experimental conditions. The influence of mutual inductance on the identification and control of SRM systems is disregarded; thus, future works should consider such influence to increase the accuracy of the proposed method.

\section{Acknowledgements}

This study was supported by the Key Scientific Study Projects of Higher Education Institutions of Henan Province (Grant Nos. 20B470003 and 18B470007) and the Promotion Special Project of Scientific Study Program of Henan Province (Grant Nos. 202102210084 and 202102210298).

This is an Open Access article distributed under the terms of the Creative Commons Attribution License

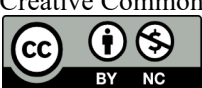

\section{References}

1. Li, S.F., Zhang, S., Habetler, T.G., Harley, R., "Modeling, design optimization, and applications of switched reluctance machines - a review". IEEE Transactions on Industry Applications, 55(3), 2019, pp. 2660-2681.
2. Bostanci, E., Moallem, M., Parsapour, A., Fahimi, B, "Opportunities and challenges of switched reluctance motor drives for electric propulsion: a comparative study". IEEE Transactions on Transportation Electrification, 3(1), 2017, pp. 58-75. 
3. Burkhart, B., Klein-Hessling, A., Ralev, I., Weiss, C., De Doncker, R., "Technology, research and applications of switched reluctance drives". CPSS Transactions on Power Electronics and Applications, 2(1), 2017, pp. 12-27.

4. Li, Y., Ma, Q., Xu, P., "Improved general modelling method of SRMs based on normalised flux linkage". Institution of Engineering and Technology Electric Power Applications, 14(2), 2020, pp. 316-324.

5. Howey, B., Bilgin, B., Emadi, A., "Design of a mutually coupled external-rotor direct drive E-bike switched reluctance motor". Institution of Engineering and Technology Electrical Systems in Transportation, 10(1), 2020, pp. 89-95.

6. Zuo, S.G., Liu, M.T., Hu S.L., "Analytical modeling for inductance and torque of switched reluctance motor considering iron core magnetic saturation". Journal of Xi'an Jiaotong University, 53(7), 2019, pp. 118-125, 143.

7. Li X., Shamsi P. "Inductance surface learning for model predictive current control of switched reluctance motors". IEEE Transactions on Transportation Electrification, 1(3), 2015, pp. 287-297.

8. Ye, W., Ma, Q.S., Xu, P., Zhang, B.M., "Nonlinear fitting method for torque-angle characteristic model of switched reluctance motor". Journal of Beijing University of Aeronautics and Astronautics, 45(1), 2019, pp. 83-92.

9. Odhano, S.A., Pescetto, P., Awan, H.A.A., Hinkkanen, M., "Pellegrino G., Bojoi R. Parameter identification and selfcommissioning in AC motor drives: a technology status review". IEEE Transactions on Power Electronics, 34(4), 2019, pp. 36033614.

10. Xu, P., Xiao, J., Li, S., Peng, X.F., "Forgetting factor based stochastic gradient identification for permanent magnet synchronous motor". Small and Special Electrical Machines, 42(4), 2014, pp. 1-3, 7.

11. Qu, B., Sun, X.F., Zhang, X.H., Huang, W., Su, J., Du, S.H., Zhai, Q.Z., Sun, R.N., Lou, Z.Y., "Correction model of energy consumption evaluation of cluster motor system based on levenberg - marquardt algorithm". Transactions of the Chinese Society of Agricultural Engineering, 34(18), 2018, pp. 44-50.

12. Accetta, A., Cirrincion,e M., Pucci, M., Sferlazza, A. "State spacevector model of linear induction motors including end - effects and iron losses PartII: model identification and results". IEEE Transactions on Industry Applications, 56(1), 2020, pp. 245-255.

13. Fagiano, L., Lauricella, M., Angelosante, D., Ragaini, E., "Identification of induction motors using smart circuit breakers". IEEE Transactions on Control Systems Technology, 27(6), 2019, pp. 2638-2646.

14. Zhang, L.W., Zhang, P., Liu, Y.F., Zhao, C., Liu, J., "Parameter identification of permanent magnet synchronous motor based on variable step - size Adaline neural network". Transactions of China Electrotechnical Society, 33(S2), 2018, pp. 377-384.
15. Wang, X.F., Lin, F., Fang, X.C., Zhang, X.Y., Yaang, Z.P., "Delay compensation for digital control system of permanent-magnet synchronous traction motor based on multiple sampling observers". Journal of the China Railway Society, 41(9), 2019, pp. 67-73.

16. Pan, Y.D., Wang, G.F., "Decoupling current control scheme for induction motors based on neutral system theory". Control and Decision, 35(2), 2020, pp. 329-338.

17. Jeon, H., Lee, J., Han, S., Kim, J.H., Hyeon, C.J., Kim, H.M., Kang, H., Ko, T.K., Yoon, Y.S., "PID control of an electromagnetbased rotary HTS flux pump for maintaining constant field in HTS synchronous motors". IEEE Transactions on Applied Superconductivity, 28(4), 2018, pp. 1-5.

18. Angel, L., Viola, J., "Design and statistical robustness analysis of FOPID, IOPID and SIMC PID controllers applied to a motor generator system". IEEE Latin America Transactions, 13(12), 2015, pp. 3724-3734.

19. Nguyen, A.T., Rafaq, M.S., Choi, H.H., Jung, J., "A model reference adaptive control - based speed controller for a surface mounted permanent magnet synchronous motor drive". IEEE Transactions on Industrial Electronics, 65(2), 2018, pp. 9399-9409.

20. Tang, C., Dai, Y., Xiao, Y., "High precision position control of PMSLM using adaptive sliding - mode approach". Journal of Electrical Systems, 10(4), 2014, pp. 456-464.

21. Nihad, A., Ateeq, U.R., Waqar, A., Hamid, M., "Disturbance observer based robust sliding mode control of permanent magnet synchronous motor". Journal of Electrical Engineering and Technology, 14(6), 2019, pp. 2531-2538.

22. Abdelkader, H., Houcine, B., Ilhami, C., Korhan, K., "Backstepping control of a separately excited DC motor". Electrical Engineering, 100(3), 2018, pp, 1393-1403.

23. Tang, C.S., Duan, Z.Y., "Direct thrust-controlled PMSLM servo system based on back-stepping control". Institute of Electrical Engineers of Japan Transactions on Electrical and Electronic Engineering, 13(5), 2018, pp. 785-790.

24. Mohamed, C., Amar, G., Med, T.B., Noureddine, G., "Sensorless finite - state predictive torque control of induction motor fed by four - switch inverter using extended Kalman filter". Compel, 37(6), 2018, pp. 2006-2024.

25. Masoudi, S., Soltanpour, M.R., Abdollahi, H., “Adaptive fuzzy control method for a linear switched reluctance motor". Institution of Engineering and Technology Electric Power Applications, 12(9), 2018, pp. 1328-1336.

26. Tang, C.S., Li, Z.M., Li, C., "Disturbance compensation based robust sliding - mode tracking control for uncertain robots". Modular Machine Tool and Automatic Manufacturing Technique, (7), 2016, pp. 99-101,104.

27. Michael, M., "Tracking and parameter identification for model reference adaptive control". International Journal of Robust and Nonlinear Control, 30(4), 2020, pp. 1582-1606. 